\title{
Frauenfragen sind Männerfragen sind Geschlechterfragen? 40 Jahre Neue Frauenbewegung. - Und jetzt?
}

\author{
Konferenz vom 12. bis 14. Juni 2008 an der Ruhr-Universität Bochum
}

Zum 40-Jährigen 'Jubiläumı der Neuen Frauenbewegung trafen sich in Bochum zwischen dem 12. und 14. Juni 2008 über 120 StudentInnen, PraktikerInnen und WissenschaftlerInnen, um Frage der Innovationskraft und Selbstreflexion der Neuen Frauenbewegung sowie deren Wirkung auf Gesellschaft, Politik und Wirtschaft zu diskutieren. Dabei wurde Wert darauf gelegt Männer mit ihren seitdem möglicherweise veränderten Lebenssituationen einzubeziehen, weil die Geschichte der Frauenbewegung in den vergangenen 40 Jahren in einem Dialog zwischen den Geschlechtern verhandelt werden sollte.

Die Hauptschwerpunkte der Konferenz waren: 1) Transformation, Selbstreflexion und Innovation der Neuen Frauenbewegung in Deutschland; 2) Politische Forderungen und Ergebnisse der Neuen Frauenbewegung in der EU-Politik; 3) Globalisierung und Frauenbewegung; 4) Zukunft der Frauenbewegung und des feministischen Denkens. Dazu stellten während der drei Tage WissenschaftlerInnen ihre neusten Forschungsergebnisse vor. In sieben kleineren Arbeitsgruppen wurde zudem ein breites Spektrum an aktuellen Fragen der Frauenbewegung und der Geschlechterpolitik behandelt.

Ilse Lenz führte mit ihrem Eröffnungsvortrag in die Konferenz ein. Sie zeigte anhand der Auswertung einer Ereignisdatenbank, in der wichtige Ereignisse der Neue Frauenbewegung in einem Zeitdiagramm aufgestellt wurden, auf, dass das immer wieder prophezeite "Ende der Frauenbewegung" ein Trugbild ist. Ihrer Darstellung nach lassen sich historisch gesehen vier Transformationen der neuen Frauenbewegung ausmachen, bei denen die Selbstreflektion der Bewegung eine wichtige Rolle spielt. Ausgangspunkt war die Bewusstwerdung und Artikulation der Neuen Frauenbewegung (1968-1976). Sie begann 1968 mit dem berühmten Tomatenwurf von Sigrid Rüger und wandelte sich 1976 mit dem Aufkommen des radikalen Gleichheitsfeminismuses in eine zweite Phase der Pluralisierung und Konsolidierung, welche 1980 in eine dritte Phase der Professionalisierung und institutionellen Integration überging, seit 1989 ist ein Wandel der Neuen Frauenbewegung hin zur Internationalisierung, Vereinigung und Neuorientierung zu beobachten. Diese neue Phase befindet sich zwischen einer hinter Familienpolitik und Globalisierungstendenzen zurücktretenden Geschlechterpolitik und Wiederaufkeimen geschlechterzentrierter Themen, welche sich durch Publikationen von jungen AutorInnen ein Sprachrohr schafft (bspw. F-Klasse, Feuchtgebiete).

$R$ alf Puchert setzte sich der Frage "Männer in Bewegung? « auseinander und zeichnete seinerseits drei Entwicklungsphasen der "Männer in Bewegung" nach. Die erste Phase beginnt mit der Entstehung der Männergruppenszene, die zweite Phase mit der Etablierung von Männerbüros, Männerforschung, kirchlicher Männerarbeit und der Männergesundheitsbewegung und die letzte Phase ist gekennzeichnet durch eine Kooperation zwischen Männern und Frauen. Abschließend verwies Puchert auf die Wandelbarkeit des sozialen Geschlechts und deutete auf ein wichtiges 
zukünftiges Feld, die Männlichkeitspolitik, hin.

In der anschließenden Diskussion wurde thematisiert, dass das Groß der Männer auch weiterhin trotz äußerlicher Offenheit, wie bereits von Ulrich Beck beschrieben, einer nur langsam schwindenden Verhaltensstarre unterliegen.

Die Beiträge von Alison Woodward und Anna Holz beleuchteten verschiedene Aspekte der politischen Forderungen und Ergebnisse der Neuen Frauenbewegung in der EU-Politik.

Woodward zeichnete in ihrem Beitrag wichtige EU-politische Veränderungen nach. Sie zeigte, dass Frauenpolitik eine wichtige Komponente der Europapolitik geworden ist und fokussierte, dass die Chancengleichheit der Frauen gerade international ausgeweitet werden muss. $\mathrm{Zu}$ dem machte sie darauf aufmerksam das der Diversity Diskurs von den zentralen Geschlechterfragen in der Europapolitik ablenkt.

In ihrem Vortrag "Entwicklung der EUGleichstellungspolitik im Prozess der europäischen Integration" knüpfte $\mathrm{Holz}$ an die historische Entwicklung der EU-Gleichstellungspolitik an.

Als Ausblick verwies Holz auf die Themen: Bekämpfung der Ungleichheit durch steuer- und sozialpolitische Maßnahmen (Gender-Budgeting), die Festzuschreibung des Frauenanteils in allen entscheidenen Gremien auf der EU-Ebene, sowie bessere Informations- und Bildungsmaßnahmen als nationales Bündnis von Wissenschaft, Politik und Zivilgesellschaft. Sie warnte vor dem Rückgang von Frauenfragen infolge von Diversity und Antidiskriminierungsmaßnahmen. Sie forderte eine stärkere Vernetzung, sowie einen europäischen Zusammenschluss von Gleichstellungsbeauftragten. Holz stellte fest, dass in Hinblick auf die EU-Gleichstellungspolitik ein neuer Feminismus wichtig ist.
Wang Zheng beschrieb in ihrem Beitrag "Global Concepts, Local Practices: Chinese Feminism since the Fourth UN Conference on Woman" den Einfluss des globalen Feminismus auf die chinesische Frauenbewegung. Trotz deutlicher historischer Unterschiede in der Entwicklung der chinesischen Frauenbewegung hat es in China fruchtbare Ansätze für ein Empowerment von Frauen für den Bereich des Gender Mainstreaming gegeben. Die Frauen konnten sich einen Raum für Diskussionen im Staatssystem schaffen. An ihrer Herkunftsuniversität, der University of Michigan, besteht ein Global Feminisms Project, das nicht nur Kontakte mit chinesischen Frauen und Frauengruppen, sondern auch mit solchen aus anderen Weltgegenden unterhält (bspw. www.umich. edu/ glbfem/index.html). Zheng wies darauf hin, dass dort im Juni 2009 eine Konferenz zu internationalen Frauenfragen stattfinden wird

In den sieben verschiedenen Arbeitsgruppen ging es um die Themen: "Das Wechselverhältnis von autonomer und institutionalisierter Frauenbewegung in der Geschlechterpolitik", "Versorgungsarbeit - Who cares? - Care und neue Arbeitskonzepte im Beruf", "Alternative Lebensformen und Alltagssolidarität in der Versorgungsarbeit $"$, Die Frauenbewegung an der Arbeit" (Berufliche Qualifikation und Laufbahnen in der betrieblichen Gleichstellung, Frauenbewegungen in den $\mathrm{Ge}-$ werkschaften), "Lesben - Vom lesbischen Identitätsfeminismus zur queeren Lebensund Liebeskombinatorik", "Grenzen und Gemeinsamkeiten - Migration und Frauenbewegung", "Mehrebenenspiele - Frauenbewegungen und die EU«, "Globalisierung und die Neuen Frauenbewegungen". Übereinstimmend wurde die Notwendigkeit einer stärkeren Vernetzung thematisiert, neue Männlichkeitsbilder und Lebensentwürfe skeptisch überdacht, sowie kontrovers diskutiert und die zukünftige 
internationale Relevanz der Frauenbewegungen fokussiert.

Gudrun-Axeli Knapp und Paula-Irene Villa setzten sich in ihren Beiträgen wissenschaftskritisch mit dem zunehmenden Verlust einer gesellschaftskritischen Perspektive in der Geschlechterforschung auseinander. Sie stellten die Unschärfen in der Außenwahrnehmung von Frauenforschung und Gleichstellungsarbeit, die Abwertung der Queer Studies als "dubiose Minderheitenwissenschaften", die zunehmende Abhängigkeit der Genderforschung von wirtschaftlichen Faktoren, sowie die Selbstverständlichkeit der Gleichheitswahrnehmung von jungen Frauen kritisch heraus. Die Zukunft des feministischen Denkens beschreiben beide in der Intersektionalität des Fachgebietes. Sie fordern von den Hochschulen ein zeit- und wirtschaftsunabhängiges wissenschaftliches Arbeiten, um dem Reputationsverlust entgegen zu wirken, sowie einen größeren Austausch zwischen Wissenschaft und Praxis.

In einer anschließenden Podiumsdiskussion zum Konferenzthema setzten sich PolitikerInnen, WissenschaftlerInnen und PraktikerInnen mit der Zukunft der Geschlechterfragen auseinander. Sie themati- sierten die Nutzung von Frauennetzwerken sowie die mögliche Neuorientierung des "Neuen Mannes".

Die Beiträge zu der Konferenz »Frauenfragen sind Männerfragen sind Geschlechterfragen! 40 Jahre Neue Frauenbewegung. - Und Jetzt? ", konnten anhand vielfältiger wissenschaftlicher Beiträge und Diskussionen aus der Praxis zeigen, dass die Themen der Neuen Frauenbewegung an Brisanz nichts verloren haben. Die Debatten um Frauen-, Männer- und Geschlechterfragen sind weiterhin aktuell und werden in den verschiedenen wissenschaftlichen, politischen und praktischen Feldern behandelt. Die hohe Anzahl der Teilnehmer an dem Kongress und der über die Zeit hinausgehende Diskussionsbedarf zeigte, dass ein Dialog und eine stärkere Vernetzung zwischen Wissenschaft und Praxis von beiden Seiten nötig und erwünscht ist.

Die Konferenz an der Ruhr-Universität wurde von der Marie-Jahoda Gastprofessur für internationale Geschlechterforschung, vom Gunda-Werner-Institut für Feminismus und Geschlechterdemokratie, der Heinrich Böll Stiftung, der HeinrichBöll-Stiftung-NRW und dem Netzwerk Frauenforschung NRW veranstaltet. 